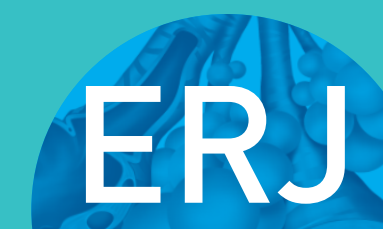

open research
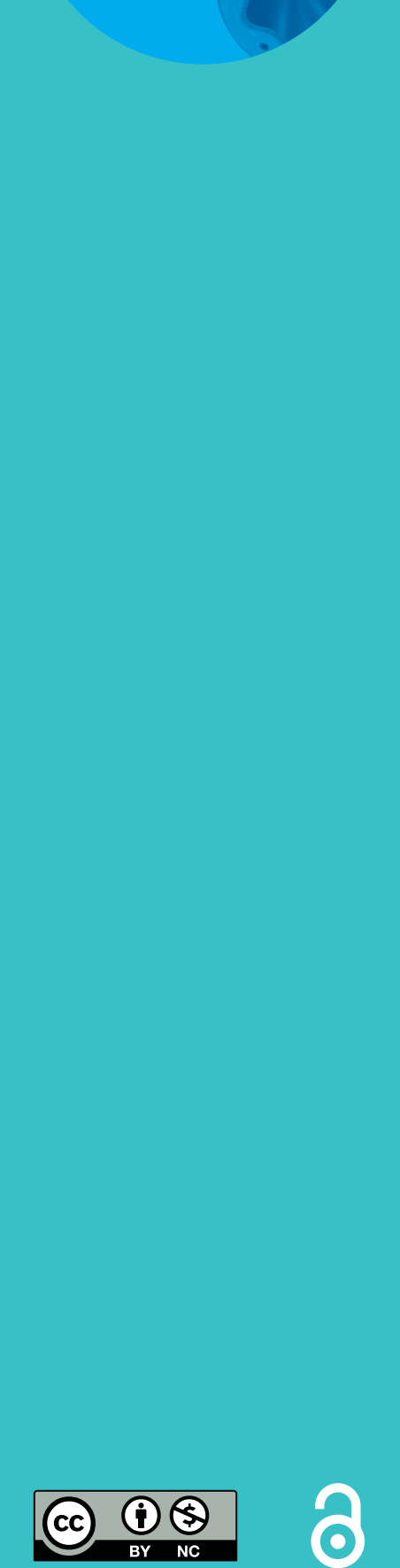

\section{Comparative assessment of several automatic CPAP devices' responses: a bench test study}

\author{
Valentina Isetta ${ }^{1,2}$, Daniel Navajas ${ }^{1,2,3}$, Josep M. Montserrat ${ }^{2,4,5}$ and \\ Ramon Farré ${ }^{1,2,5}$
}

Affiliations: ${ }^{1}$ Unitat de Biofísica i Bioenginyeria, Facultat de Medicina, Universitat de Barcelona, Barcelona, Spain. ${ }^{2}$ CIBERES, Madrid, Spain. ${ }^{3}$ Institute for Bioengineering of Catalonia (IBEC), Barcelona, Spain. ${ }^{4}$ Sleep Lab, Pneumology Department, Hospital Clinic, Barcelona, Spain. ${ }^{5}$ IDIBAPS (Institut d'investigacions Biomèdiques August Pi i Sunyer), Barcelona, Spain.

Correspondence: Ramon Farré, Unitat de Biofísica i Bioenginyeria, Facultat de Medicina - Universitat de Barcelona, Casanova 143, 08036, Barcelona, Spain. E-mail: rfarređub.edu

ABSTRACT Automatic continuous positive airway pressure (APAP) devices adjust the delivered pressure based on the breathing patterns of the patient and, accordingly, they may be more suitable for patients who have a variety of pressure demands during sleep based on factors such as body posture, sleep stage or variability between nights. Devices from different manufacturers incorporate distinct algorithms and may therefore respond differently when subjected to the same disturbed breathing pattern. Our objective was to assess the response of several currently available APAP devices in a bench test.

A computer-controlled model mimicking the breathing pattern of a patient with obstructive sleep apnoea (OSA) was connected to different APAP devices for 2-h tests during which flow and pressure readings were recorded. Devices tested were AirSense 10 (ResMed), Dreamstar (Sefam), Icon (Fisher \& Paykel), Resmart (BMC), Somnobalance (Weinmann), System One (Respironics) and XT-Auto (Apex). Each device was tested twice.

The response of each device was considerably different. Whereas some devices were able to normalise breathing, in some cases exceeding the required pressure, other devices did not eliminate disturbed breathing events (mainly prolonged flow limitation). Mean and maximum pressures ranged 7.3$14.6 \mathrm{cmH}_{2} \mathrm{O}$ and $10.4-17.9 \mathrm{cmH}_{2} \mathrm{O}$, respectively, and the time to reach maximum pressure varied from 4.4 to $96.0 \mathrm{~min}$.

Each APAP device uses a proprietary algorithm and, therefore, the response to a bench simulation of OSA varied significantly. This must be taken into account for nasal pressure treatment of OSA patients and when comparing results from clinical trials.

@ERSpublications

There are considerable differences between APAP devices subjected to simulated OSA http://ow.ly/QtmxI

Received: June 052015 | Accepted after revision: July 262015

Support statement: This work was carried out within the framework of a ResMed-University of Barcelona contract aimed at bench testing automatic continuous positive airway pressure devices. ResMed provided financial support to carry out the study but did not intervene in the design or performance of the tests, or in the data analysis. ResMed also funded the assistance of a medical writer in preparing the manuscript. Funding information for this article has been deposited with FundRef.

Conflict of interest: Disclosures can be found alongside this article at openres.ersjournals.com

Copyright $\odot$ ERS 2015. This article is open access and distributed under the terms of the Creative Commons Attribution Non-Commercial Licence 4.0. 


\section{Introduction}

Continuous positive airway pressure (CPAP) is the treatment of choice for patients with obstructive sleep apnoea (OSA), regardless of disease severity $[1,2]$. CPAP has been shown to decrease elevated blood pressure, improve cardiovascular disease outcomes, and reduce the risk of fatal and nonfatal cardiovascular events [3-5]. It also improves excessive daytime sleepiness and restores impaired cognitive function [6].

Adherence to CPAP therapy is necessary for achieving satisfactory treatment outcomes [7]. However, CPAP compliance tends to be suboptimal, both in terms of treatment acceptance and hours of CPAP use per night, with compliance reported to be as low as 30-60\% [8]. Although therapy acceptance depends on patient characteristics, equipment-related factors are crucial in determining CPAP adherence.

Automatic CPAP (APAP) is an alternative treatment for CPAP-intolerant OSA patients [1] and was developed to improve compliance. APAP devices reduce mean nocturnal pressure by automatically adjusting the delivered pressure based on the changing requirements of the patient [9-14]. Accordingly, APAP therapy might be more suitable for patients who have a variety of pressure demands during sleep based on factors such as body posture, sleep stage or variability between nights [15-19]. However, data on the advantage of APAP over CPAP for the general population of OSA patients remain controversial $[20,21]$. APAP does seem to be better suited for specific OSA phenotypes, although defining the optimal target population for this therapy requires further research.

Given the technical complexity of APAP engineering, each manufacturer designs its own solution to detect disturbed breathing events and potential artefacts selectively, and to define a strategy for automatic adaptation of nasal pressure. Therefore, the use of different proprietary algorithms in APAP devices usually leads to distinct responses to the same sleep-related breathing conditions. As a result, devices from different manufacturers cannot be considered equal, particularly with respect to clinical performance. Despite existing studies comparing APAP technologies, the ongoing development of more technologically advanced devices and their availability on the market means that objective analyses are required to provide reliable data on which to base clinical decisions. Thus, the aim of this study was to compare the responses of several currently available APAP devices using a bench test simulation of OSA disturbed breathing patterns.

\section{Methods}

The bench test method used in this study was described in detail previously $[22,23]$. Briefly, the bench test simulator comprised a flow generator controlled by a computer. The piston-based flow generator can reproduce mathematically designed breathing flows or replicate respiratory flows of patients recorded during polysomnography (PSG). A computer-controlled obstruction valve allows the simulation of central or obstructive events. The bench platform is equipped with two sensors (one for pressure and one for flow) and data are recorded on the computer for subsequent analysis. Conventional tubing connects the APAP device under test to the simulated OSA patient.

The disturbed breathing events employed in this study were extracted from real PSG recordings of OSA patients as previously described [22]. The simulator was set to reproduce the breathing events depending on the positive airway pressure (PAP) applied (figure 1): 1) apnoeas with obstruction for $\mathrm{PAP}<5 \mathrm{cmH}_{2} \mathrm{O}$; 2) severe hypopnoeas for PAP between 5 and $\left.7 \mathrm{cmH}_{2} \mathrm{O} ; 3\right)$ mild hypopneas for PAP between 7 and $10 \mathrm{cmH}_{2} \mathrm{O}$; 4) prolonged flow limitation for PAP between 10 and $12 \mathrm{cmH}_{2} \mathrm{O}$; and 5) normal breathing for PAP $>12 \mathrm{cmH}_{2} \mathrm{O}$. Each test started with $15 \mathrm{~min}$ of normal breathing to simulate the time before sleep onset, followed by $2 \mathrm{~h}$ of simulated OSA.

The study was performed on seven APAP devices currently in clinical use: AirSense 10 (A) manufactured by ResMed (San Diego, CA, USA), Dreamstar (B) by SEFAM (Villers-lès-Nancy, France), Icon (C) by Fisher \& Paykel (Auckland, New Zealand), Resmart (D) by BMC (Beijing, China), Somnobalance (E) by Weinmann (Hamburg, Germany), System One (F) by Respironics (Murrysville, PA, USA) and XT-Auto (G) by Apex (New Taipei City, Taiwan). For the AirSense 10 device, two different inbuilt algorithms were tested, standard (A1) and response (A2) settings, with the Expiratory Pressure Relief setting off. Each device was equipped with its corresponding tubing. The minimum and maximum pressures were set at 4 and $20 \mathrm{cmH}_{2} \mathrm{O}$, respectively. Any other programmable settings were left at their default values. Ramp time was off for all devices and no humidification was used during any of the testing. Each test was repeated twice and the corresponding average result is reported.

\section{Results}

The responses of the assessed APAP devices are summarised in table 1. There was considerable variation among devices, particularly with respect to the mean and maximum nasal pressures applied, the time to reach maximum nasal pressure, and the residual apnoea-hypopnoea index (number of residual obstructive events per hour). More than five residual obstructive events per hour were observed with devices B, D, F 
FIGURE 1 Breathing patterns reproduced by the simulated obstructive sleep apnoea patient depending on the continuous positive airway pressure (CPAP) applied by automatic CPAP devices: a) normal breathing; b) prolonged flow limitation; c) mild hypopnoea; d) severe hypopnoea; el apnoea with obstruction. Reproduced from [23] with permission from the publisher.

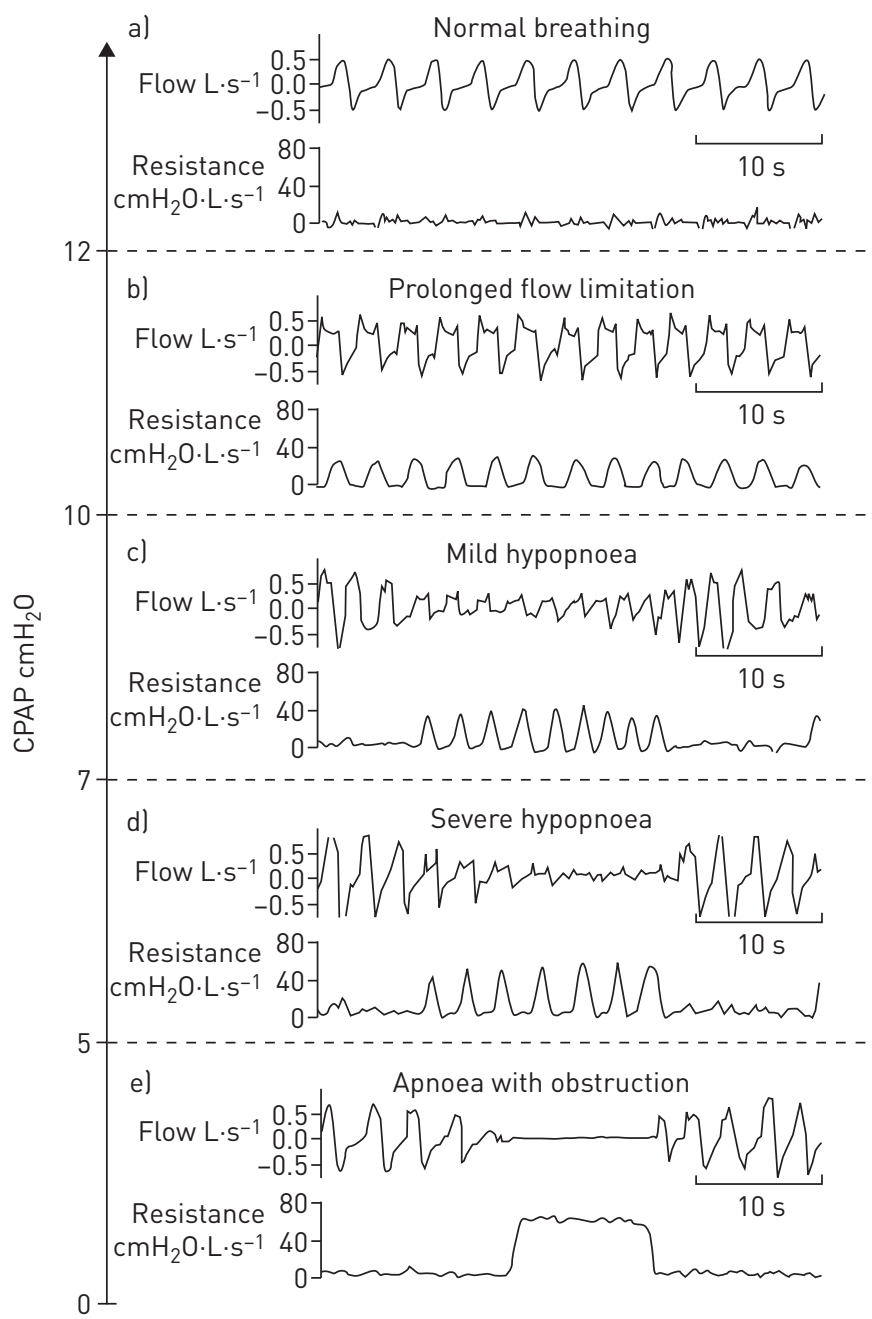

and G. Breathing normalisation (defined as the avoidance of any obstructive event: apnoea, hypopnoea or flow limitation) was only achieved with the A1, A2 and $\mathrm{C}$ devices. Figure 2 shows examples of the first $30 \mathrm{~min}$ of device response for one device that achieved breathing normalisation (A2) and another that was unable to overcome obstructive events (B).

TABLE 1 Reponses of automatic continuous positive airway pressure (APAP) devices to obstructive sleep apnoea simulated by the bench test

\begin{tabular}{|c|c|c|c|c|c|}
\hline APAP device & $P_{\max } \mathrm{cmH}_{2} \mathrm{O}$ & $t_{\max } \min$ & $P_{\text {mean }} \mathrm{cmH}_{2} \mathrm{O}$ & $\begin{array}{c}\text { Residual } \\
\text { AHI events per } h\end{array}$ & $\begin{array}{c}\text { Breathing } \\
\text { normalisation }\end{array}$ \\
\hline A1 & $17.9,17.8$ & $22.4,19.0$ & $14.6,14.6$ & $2.0,2.0$ & Yes, yes \\
\hline A2 & $15.4,15.6$ & $40.3,57.7$ & $13.4,13.5$ & $2.0,1.5$ & Yes, yes \\
\hline B & $10.5,10.5$ & $130.6,121.3$ & $6.7,7.9$ & $74.5,71.0$ & No, no \\
\hline C & $13.4,13.9$ & $28.0,44.1$ & $12.4,12.2$ & $3.0,3.0$ & Yes, yes \\
\hline D & $10.7,10.7$ & $75.1,103.7$ & $9.7,9.7$ & $26.5,32.5$ & No, no \\
\hline $\mathbf{E}$ & $10.4,10.4$ & $20.7,18.9$ & $10.2,10.2$ & $3.5,2.5$ & No, no \\
\hline $\mathbf{F}$ & $11.9,12.1$ & $34.4,36.0$ & $10.1,10.1$ & $11.5,13$ & No, no \\
\hline G & $10.5,11.0$ & $32.2,83.2$ & $9.9,9.9$ & $33,26.5$ & No, no \\
\hline
\end{tabular}

The two values for each variable correspond to the results obtained in the two test repetitions in each device. $P_{\max }$ : maximum positive airway pressure applied; $t_{\max }$ : time to reach $P_{\max } \pm 0.3 \mathrm{cmH}_{2} \mathrm{O} ; P_{\text {mean }}$ : mean positive airway pressure; AHI: apnoea-hypopnoea index; A1: AirSense 10, standard setting; A2: AirSense 10, response setting; B: Dreamstar; C: Icon; D: Resmart; E: Somnobalance; F: System One; G: XT-Auto. 
FIGURE 2 Example of the first 30-min response of two automatic continuous positive airway pressure devices: one achieving breathing normalisation (AirSense 10 response setting (A2)) and another unable to eliminate obstructive events (Dreamstar (B)). The dashed black line represents the pressure required to achieve breathing normalisation $\left(12 \mathrm{cmH}_{2} \mathrm{O}\right)$.

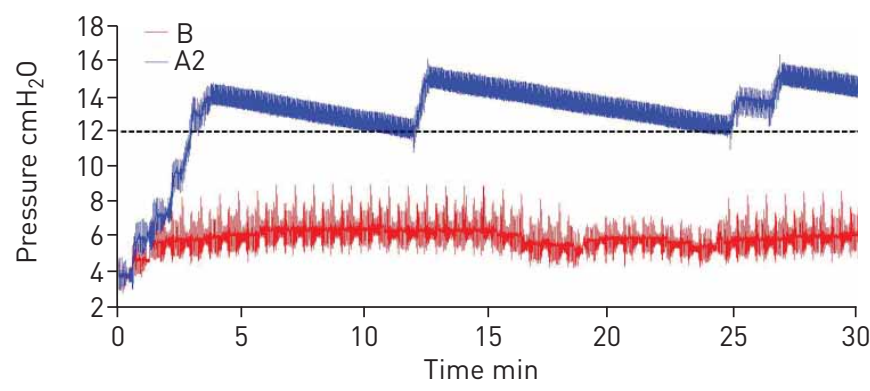

The assessed devices also showed different performances in the rate of pressure increase after occurrence of the first obstructive event. Tracings of the initial $10 \mathrm{~min}$ of the test results are shown in figure 3 . Device A1 responded to obstructive events with a step-wise increase in pressure sufficient to overcome obstruction (mean maximum pressure $17.9 \mathrm{cmH}_{2} \mathrm{O}$ ). A2 increases in pressure were more gradual but the pressures reached (mean maximum $15.5 \mathrm{cmH}_{2} \mathrm{O}$ ) were sufficient to normalise breathing. Devices $\mathrm{C}$ and $\mathrm{E}$ also showed a rapid and pronounced pressure increase after the first obstructive event, even if only device $\mathrm{C}$ reached a pressure sufficient to normalise breathing (mean maximum $13.6 \mathrm{cmH}_{2} \mathrm{O}$ ). The other devices increased pressure more slowly and none of them was able to completely normalise breathing.

\section{Discussion}

This bench test investigated the response of seven currently used APAP devices and found marked differences in their responses to a simulated breathing pattern mimicking that of an OSA patient. Only two devices were able to achieve breathing normalisation and to reach a residual $\mathrm{AHI}<5$, although the nasal pressures reached in some cases were higher than those required to normalise breathing in the simulated patient. Moreover, the rate of pressure increase in response to obstructive events was considerably different when comparing the tested APAP devices.

The findings obtained when testing currently available devices in this study are similar to those in previous reports, which showed variability in the response of APAP devices when subjected to the same breathing pattern under well-controlled conditions [22-24]. Thus, the fact that APAP devices behave differently should not be attributable to the lack of technical maturity originally found in new technologies but to the manufacturers strategy of offering devices with differentiated functioning features aimed at better treating patients. In fact, it is most likely that this inter-device response variation can be attributed to the different in-built algorithms operating in each device.

- The first step in the algorithms incorporated into APAP devices should analyse the pressure/flow data recorded by in-built transducers to identify different types of breathing events (e.g. apnoeas, hypopneas, snoring and flow limitation).

FIGURE 3 Pressure increase delivered by the tested automatic continuous positive airway pressure devices during the first $10 \mathrm{~min}$ of the bench test. The dashed black line represents the pressure required to achieve breathing normalisation $\left(12 \mathrm{cmH}_{2} \mathrm{O}\right)$. A1: AirSense 10, standard setting; A2: AirSense 10, response setting; B: Dreamstar; C: Icon; D: Resmart; E: Somnobalance; F: System One; G: XT-Auto.

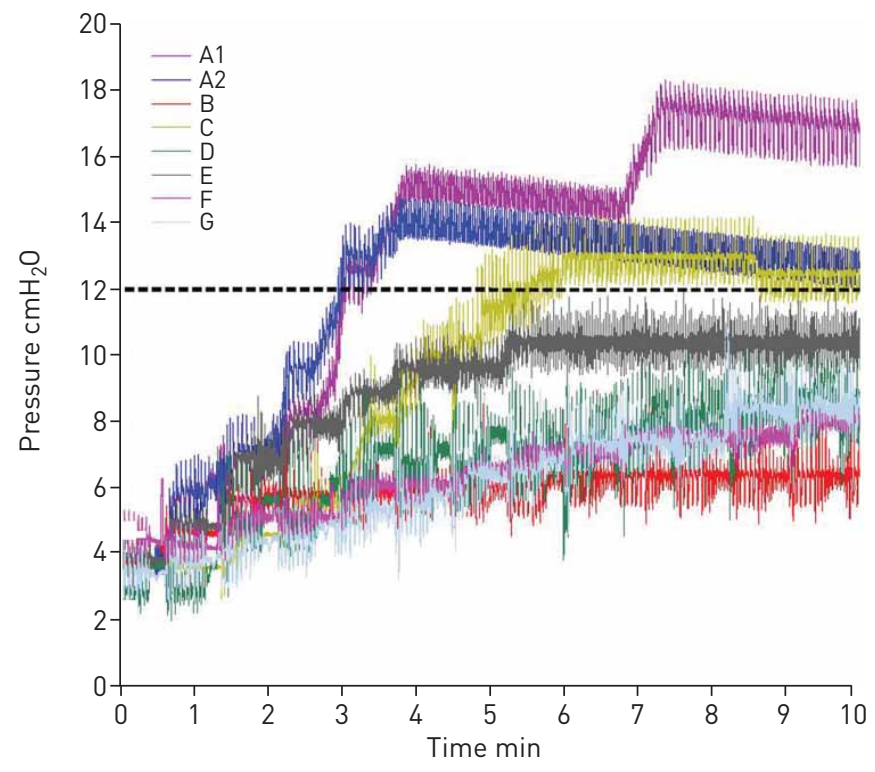


- The algorithm must also distinguish breathing events from artefacts (e.g. swallowing, coughing and speaking). Indeed, variation among devices response will depend significantly on the detection of events with no well-established definition, such as flow limitation, the detection of which remains open to interpretation. Therefore, the ability of a device to recognise and classify this event depends on the potential agreement between the specific flow limitation pattern simulated in the bench study and the event definition implemented in the APAP device algorithm.

- Once breathing events have been detected, the algorithm should decide when and for how long to modify the applied nasal pressure. The differences observed in this study were, therefore, not unexpected and do not necessarily imply incorrect device performance. For instance, the optimal rate of pressure increase after detection of obstructive events, which varied considerably between the devices tested (figure 3), is not clear. Specifically, a difficult balance needs to be found between a response that is fast enough to ensure avoidance of disturbed breathing and soft enough to avoid patient awakening in response to a sudden nasal pressure increase.

In addition, it should be noted that default settings were used during testing of most of the devices in this study and that it is possible that selecting other response thresholds could have an impact on their performance. Whether or not such modifications would increase the number of devices able to normalise breathing under the test conditions used in this study or the values of nasal pressure applied to normalise breathing is unknown.

Although bench testing is useful to understand and characterise the response of APAP devices under well-controlled conditions, this evaluation method has some limitations. Defining a model of OSA patient does not allow reproduction of the almost infinite variation in breathing events observed in clinical practice, or uncontrolled mask leaks, snoring or mouth expiration. Accordingly, it is possible that the responses of the tested devices would have been different from those reported if OSA was simulated by another model. In fact, bench testing should be considered a preliminary evaluation before the device is fully assessed in the clinical setting. However, this testing has shown that the high residual AHI seen in some devices should be further investigated in clinical practice to ensure that patients using these devices do have their sleep apnoea effectively treated. Bench test results can thus be useful when selecting the most suitable device for each patient to improve comfort and treatment compliance, and when interpreting the results of clinical studies if different devices are involved.

In conclusion, this bench study showed considerable response differences between several currently used APAP devices when subjected to a specific simulated OSA breathing pattern. Although APAP is a useful therapy, these results underline the concept that the actual implementation of APAP depends on the product-specific engineering solutions and algorithms adopted by each manufacturing company, which has implications for clinical application.

\section{Lessons for clinicians}

- This bench test study assessed how currently available APAP devices respond to a simulated OSA patient. Regardless of the simplified experimental setting employed in this work, as compared with the complexity of events found in the clinical arena when treating OSA patients, the following practical lessons can be derived.

- The way that each commercial APAP device modifies nasal pressure when subjected to disturbed breathing patterns is different.

- These differences in response among APAP devices are not necessarily caused by incorrect performances. Instead, they are the result of the particular engineering solutions implemented in each device.

- Knowing the specific functioning features of each APAP device (e.g. sensitivity in detecting the different obstructive events, tolerance to events before increasing pressure and speed of pressure changes) may help to understand treatment compliance in specific patient phenotypes.

- Choosing the optimal APAP device for the needs/preferences of each individual patient may improve therapy compliance.

\section{Acknowledgements}

The authors wish to thank Miguel Angel Rodriguez (University of Barcelona, Barcelona, Spain) for his technical support in execution of the tests and Nicola Ryan, an independent medical writer funded by ResMed, for her assistance in preparing the manuscript.

\section{References}

1 Epstein LJ, Kristo D, Strollo PJ Jr, et al. Clinical guideline for the evaluation, management and long-term care of obstructive sleep apnea in adults. J Clin Sleep Med 2009; 5: 263-276.

2 Qaseem A, Holty JE, Owens DK, et al. Management of obstructive sleep apnea in adults: a clinical practice guideline from the American College of Physicians. Ann Intern Med 2013; 159: 471-483. 
3 Buchner NJ, Sanner BM, Borgel J, et al. Continuous positive airway pressure treatment of mild to moderate obstructive sleep apnea reduces cardiovascular risk. Am J Respir Crit Care Med 2007; 176: 1274-1280.

4 Haentjens P, Van Meerhaeghe A, Moscariello A, et al. The impact of continuous positive airway pressure on blood pressure in patients with obstructive sleep apnea syndrome: evidence from a meta-analysis of placebo-controlled randomized trials. Arch Intern Med 2007; 167: 757-764.

5 Marin JM, Carrizo SJ, Vicente E, et al. Long-term cardiovascular outcomes in men with obstructive sleep apnoea-hypopnoea with or without treatment with continuous positive airway pressure: an observational study. Lancet 2005; 365: 1046-1053.

6 Engleman HM, Douglas NJ. Sleep. 4: Sleepiness, cognitive function, and quality of life in obstructive sleep apnoea/ hypopnoea syndrome. Thorax 2004; 59: 618-622.

7 Barbé F, Durán-Cantolla J, Capote F, et al. Long-term effect of continuous positive airway pressure in hypertensive patients with sleep apnea. Am J Respir Crit Care Med 2010; 181: 718-726.

8 Weaver TE, Sawyer AM. Adherence to continuous positive airway pressure treatment for obstructive sleep apnoea: implications for future interventions. Indian J Med Res 2010; 131: 245-258.

9 Ayas NT, Patel SR, Malhotra A, et al. Auto-titrating versus standard continuous positive airway pressure for the treatment of obstructive sleep apnea: results of a meta-analysis. Sleep 2004; 27: 249-253.

10 Behbehani K, Yen FC, Lucas EA, et al. A sleep laboratory evaluation of an automatic positive airway pressure system for treatment of obstructive sleep apnea. Sleep 1998; 21: 485-491.

11 d'Ortho MP, Grillier-Lanoir V, Levy P, et al. Constant vs. automatic continuous positive airway pressure therapy: home evaluation. Chest 2000; 118: 1010-1017.

12 Massie CA, McArdle N, Hart RW, et al. Comparison between automatic and fixed positive airway pressure therapy in the home. Am J Respir Crit Care Med 2003; 167: 20-23.

13 Randerath WJ, Galetke W, Ruhle KH. Auto-adjusting CPAP based on impedance versus bilevel pressure in difficult-to-treat sleep apnea syndrome: a prospective randomized crossover study. Med Sci Monit 2003; 9 : CR353-CR358.

14 Teschler H, Wessendorf TE, Farhat AA, et al. Two months auto-adjusting versus conventional nCPAP for obstructive sleep apnoea syndrome. Eur Respir J 2000; 15: 990-995.

15 Marrone O, Insalaco G, Bonsignore MR, et al. Sleep structure correlates of continuous positive airway pressure variations during application of an autotitrating continuous positive airway pressure machine in patients with obstructive sleep apnea syndrome. Chest 2002; 121: 759-767.

16 Noseda A, Kempenaers C, Kerkhofs M, et al. Constant vs auto-continuous positive airway pressure in patients with sleep apnea hypopnea syndrome and a high variability in pressure requirement. Chest 2004; 126: 31-37.

17 Oksenberg A, Silverberg DS, Arons E, et al. The sleep supine position has a major effect on optimal nasal continuous positive airway pressure: relationship with rapid eye movements and non-rapid eye movements sleep, body mass index, respiratory disturbance index, and age. Chest 1999; 116: 1000-1006.

18 Penzel T, Moller M, Becker HF, Effect of sleep position and sleep stage on the collapsibility of the upper airways in patients with sleep apnea. Sleep 2001; 24: 90-95.

19 Series F, Marc I. Importance of sleep stage- and body position-dependence of sleep apnoea in determining benefits to auto-CPAP therapy. Eur Respir J 2001; 18: 170-175.

20 Woehrle H, Graml A, Weinreich G. Age- and gender-dependent adherence with continuous positive airway pressure therapy. Sleep Med 2011; 12: 1034-1036.

21 Ip S, D'Ambrosio C, Patel K, et al. Auto-titrating versus fixed continuous positive airway pressure for the treatment of obstructive sleep apnea: a systematic review with meta-analyses. Syst Rev 2012; 1: 20.

22 Farré R, Montserrat JM, Rigau J, et al. Response of automatic continuous positive airway pressure devices to different sleep breathing patterns: a bench study. Am J Respir Crit Care Med 2002; 166: 469-473.

23 Rigau J, Montserrat JM, Wöhrle $\mathrm{H}$, et al. Bench model to simulate upper airway obstruction for analyzing automatic continuous positive airway pressure devices. Chest 2006; 130: 350-361.

24 Abdenbi F, Chambille B, Escourrou P. Bench testing of auto-adjusting positive airway pressure devices. Eur Respir J 2004; 24: 649-658. 